\title{
Liraglutide \& dulaglutide in addition to SGLT-2i and metformin in Indian T2DM: a retrospective observational study
}

\section{S. Ghosal' ${ }^{1}$ B. Sinha ${ }^{2}$.}

${ }^{1}$ Nightingale Hospital, Endocrinology, Kolkata, India.

${ }^{2} \mathrm{AMRI}$ hospitals, Endocrinology, Kolkata, India.

\section{ABSTRACT:}

Aim: The aim of this analysis was to test the additive metabolic benefit of sequential addition of SGLT-2i followed by GLP1-RA along with metformin in a real-life scenario

Material \& Methods: A retrospective real world observational case note study conducted in two diabetes care centres in India analyzed the first 60 consecutive T2D patients who could afford this therapy and had not achieved their glycaemic target on metformin and SGLT2i were added either Dulaglutide or Liraglutide and followed up for 13 weeks.

Results: Both liraglutide and dulaglutide proved to be an excellent add on to metformin and SGLT-2 inhibitor. Liraglutide had an additional significant impact on systolic blood pressure reduction as well as larger proportion
achieved target HBA1C in contrast to the dulaglutide.

Conclusion: In this retrospective study in Indian type 2 diabetic patients poorly controlled with metformin and SGLT-2 inhibitor we found a meaningful impact of adding a GLP1-RA on all metabolic parameters. There were additional advantages seen with liraglutide as far achieving target $\mathrm{HBA1C}$ of less than $7 \%$ and also on the quantum of weight loss and systolic blood pressure reduction.

Keywords:

Key Messages:

\begin{tabular}{l}
\hline I. This is the first real life data on metabolic impact of sequential addition of GLP1-RA after failure of metformin \\
and SGLT-2 inhibitor. \\
II. This study demonstrated a significant metabolic benefit of adding either dulaglutide or liraglutide after \\
metformin and SGLT-2 inhibitor failure. \\
III. This study also demonstrated superior reduction of weight and systolic blood pressure with liraglutide \\
compared to dulaglutide with larger proportion of patients achieving HBA1C targets in liraglutide arm. \\
IV. The prime importance of this study is related to the fact that in India patients have today out of their pocket for \\
medications.
\end{tabular}

\section{Introduction:}

The combination of Glucagon like Peptide Receptor Analogues (GLP-1RA) with a Sodium-glucose linked transporter inhibitor (SGLT2i) addresses six out of the eight ominous pathophysiological defects seen in Type 2 Diabetes (T2D). (1). Combining both these class of drugs seems to address the pathophysiologic
$(2,3,4,5)$. Hence there is a good scientific rationale for using them in combination.

The recent DURATION 8 study, testing the above rationale, demonstrated an additive effect on weight and systolic the $1 \mathrm{C}$ reduction when exenatide LAR and dapagliflozin were used in combination as a co-initiation strategy (6).

In a resource poor setting such as India where patients pay 'out-of-pocket', injectable therapies are usually used as a third-line agent when oral therapy fails. Hence, data on this combination mimicking a real-life setting i.e. oral therapy followed by injectable woun

Materials \& Methods:

A retrospective, real world observational study to evaluate the efficacy of triple-anti-hyperglycemic agent therapy namely metformin, sodium glucose co-transporter 2 Inhibitors (SGLT-2Is) and glucagon like peptide receptor agonists the outpatients of two hospitals in Kolkata, India, from May 2016 to August 2016. The baseline characteristics of the patients included for analysis are detailed in table 1 (Supplementary appendix).

After clearance from the local ethics committee, the case notes of the first 30 consecutive patients who had been commenced on Metformin plus SGLT2i plus Dulaglutide plus the first 30 patients who had been commenced on
Metformin plus SGLT2i plus Liraglutide was collated.

After adequate counseling patients who could afford this expensive combination of medications for at least 3 months had been commenced on this therapy on the basis of the following inclusion and exclusion criteria:

Inclusion Criteria:

Adult Type 2 diabetics with $\mathrm{HbA1C} \geq 7.0 \%$ on Metformin plus SGLT2

$\mathrm{BMI} \geq 25 \mathrm{~kg} / \mathrm{m}^{2}$,

Exclusion Criteria:

Type 1 Diabetes

Pregnancy

Deranged liver function tests

Any major organ system

Recent insulin therapy

Recent anti obesity therapy

Recent treatment with any other oral anti diabetics

Patients received treatment as per routine standard of care. All anti hypertensives, anti hyperlipidaemics and anti platelet agents and other preexisting medications were continued as per the patients' requirements. All patients' records with respect to age, gender, height, body weight, BMI, duration of diabetes, HbAlc, FPG, BP and adverse effects were collected from the case note database. Blood glucose was measured by hexokinase method and HbAlc was me
high performance liquid chromatographic (HPLC) method (Bio-RAD D-10, Bio-RAD, Hercules, CA, USA).

All 30 patients had been maintained on Liraglutide in a dose of $1.2 \mathrm{mg}$ per day and all 30 patients on dulaglutide $1.5 \mathrm{mg}$ dose once-weekly during the thirteen weeks of the study period. Both the arms were well matched as far as baseline characteristics were concerned. (Table 2 - Supplementary appendix).

Statistical Methods:

Descriptive statistical analysis were carried out with a Statistical software named SAS (Statistical Analysis System) version 9.2 for windows, SAS Institute Inc. Cary, NC, USA and Statistical Package for Social Sciences (SPSS Complex
Samples) Version 21.0 for windows, SPSS, Inc., Chicago, IL, USA, with Microsoft Word and Excel being used to Samples) Version 21.0 for windows, SPSS, Inc., Chicago, IL, USA, with Microsoft Word and Excel being used to
generate graphs and tables. Results on continuous measurements are presented as Mean + SEM and results on generate graphs and tables. Results on continuous measurements are presented as Mean \pm
categorical measurements are presented in Number (\%). Significance is assessed at a level of $5 \%$.

The following assumptions on data were made: 1) Cases of the samples should be independent, 2) The populations from which the samples are drawn have the same variance (or standard deviation) and 3) The samples drawn from different which the samples are

Normality of data was tested by Anderson Darling test, Shapiro-Wilk, Kolmogorov-Smirnoff test and visually by QQ plot. Paired t-test was used to find the significance of study parameters within groups of patients measured on two
occasions. Chi-square/ Fisher Exact test has been used to find the significance of study parameters on categorical scale between two or more groups.

Results:

Impact of adding either Dulaglutide or Liraglutide to Metformin and SGLT2 Inhibitors:

Dulaglutide:

Adding dulaglutide to the combination of metformin $2000 \mathrm{mg} /$ day and aSGLT2i resulted in a significant reduction in FPG $(-41.87 \pm 12.72 \mathrm{mg} / \mathrm{dL} ; \mathrm{p}=0.003)$ and $\mathrm{HBA1C}$ at 3 months follow up $(-1.017 \pm 0.22 \%$; $\mathrm{p}<<0.001)$. [Table 1$]$ The
impressive reduction in glycemic parameters was accompanied by a significant impact on body weight $(-4.20 \pm 0.47 \mathrm{~kg}$; $\mathrm{p}<<0.001$ and $\mathrm{BMI}(-1.53 \pm 0.21 \% ; \mathrm{p}<0.001)$ without an impact on BP.

Liraglutide:

As with dulaglutide, treatment with liraglutide in addition to Metformin and a SGLT2i resulted in a significant reduction in FPG $(-44.20 \pm 8.05 \mathrm{mg} / \mathrm{dL} ; \mathrm{p}<0.001)$, HBA1C $(1.547 \pm 0.22 \%$; $<<0.001)$, weight $(-5.83 \pm 0.87 \mathrm{~kg} ; \mathrm{p}<0.001)$ and BMI $(2.27 \pm 0.33 \& ; \mathrm{p}<0.001)$ at 3 months follow up. [Table 3 ] However in contrast to
with a significant reduction in $\operatorname{SBP}(-10.23 \pm 2.36 \mathrm{~mm}$ of $\mathrm{Hg} ; \mathrm{p}<0.001)$. [Table 1 ]
Dulaglutide versus Liraglutide failing $\mathrm{OHA}$ regime:

Both dulaglutide and liraglutide were independently associated with a significant impact on HBA1C and weight with liraglutide having an additional impact on SBP. We also analyzed the data to compare the effects of dulaglutide with Glycemic Parameters:

Compared to dulaglutide, liraglutide was associated with a non-significantly greater reduction in both FPG (-44.2 \pm 8.05 $\mathrm{mg} / \mathrm{dL}$ with liraglutide versus $-41.87 \pm 12.72 \mathrm{mg} / \mathrm{dL}$ with dulaglutide) and $\mathrm{HBA} 1 \mathrm{C}(-1.55 \pm 0.22$ with Liraglutide versus
$-1.02 \pm 0.22$ with dulaglutide $)(\mathrm{p}=0.091)$. [Table 3 ] However, $63.3 \%$ of patients in the liraglutide achieved an HBA1C $-1.02 \pm 0.22$ with dulaglutide) ( $\mathrm{p}=0.091)$. [Table 3 ] However, $63.3 \%$ of patients in the liraglutide achieved an HBA1C of $<7.0 \%$ compared to only
Supplementary appendix]

\begin{tabular}{|c|c|c|c|c|c|c|c|c|}
\hline \multicolumn{9}{|c|}{ TABLE 3- Change in study parameters during the follow-up period } \\
\hline Cohort & \multicolumn{4}{|c|}{ Dulaglutide, $\mathbf{n = 3 0}$} & \multicolumn{4}{|c|}{ Liraglutide, $n=30$} \\
\hline Parameter & $\begin{array}{c}\text { Baseline } \\
\text { Mean } \pm \text { SEM }\end{array}$ & $\begin{array}{c}\text { Follow-up } \\
\text { Mean } \pm \text { SEM }\end{array}$ & $\begin{array}{c}\text { Change Mean } \\
\pm \text { SEM }\end{array}$ & $\mathrm{P}$ & $\begin{array}{c}\text { Baseline } \\
\text { Mean } \pm S E M\end{array}$ & $\begin{array}{c}\text { Follow-up } \\
\text { Mean } \pm \text { SEM }\end{array}$ & $\begin{array}{c}\text { Change Mean } \\
\pm \text { SEM }\end{array}$ & $\mathrm{P}$ \\
\hline $\begin{array}{l}\text { Body weight } \\
(\mathrm{kg})\end{array}$ & $\begin{array}{r}87.17 \\
\pm 2.16\end{array}$ & $\begin{array}{r}82.97 \\
\pm 2.05\end{array}$ & $\begin{array}{r}-4.20 \\
\pm 0.47\end{array}$ & $<0.001$ & $\begin{array}{r}89.43 \\
\pm 2.60\end{array}$ & $\begin{array}{r}83.60 \\
\pm 2.35\end{array}$ & $\begin{array}{r}-5.83 \\
\pm 0.87\end{array}$ & $<0.001$ \\
\hline $\mathrm{BMI}\left(\mathrm{kg} / \mathrm{m}^{2}\right)$ & $\begin{array}{r}32.84 \\
\pm 0.77 \\
\end{array}$ & $\begin{array}{r}31.312 \\
\pm 0.68 \\
\end{array}$ & $\begin{array}{r}-1.53 \\
\pm 0.21 \\
\end{array}$ & $<0.001$ & $\begin{array}{r}34.92 \\
\pm 0.868 \\
\end{array}$ & $\begin{array}{r}32.650 \\
\pm 0.77 \\
\end{array}$ & $\begin{array}{r}-2.27 \\
\pm 0.33 \\
\end{array}$ & $<0.001$ \\
\hline $\mathrm{SBP}(\mathrm{mmHg})$ & $\begin{array}{r}134.30 \\
\pm 2.71\end{array}$ & $\begin{array}{r}130.87 \\
\pm 2.49\end{array}$ & $\begin{array}{r}-3.43 \\
\pm 2.97\end{array}$ & 0.258 & $\begin{array}{r}137.83 \\
\pm 2.503\end{array}$ & $\begin{array}{l}127.60 \\
\pm 2.07\end{array}$ & $\begin{array}{l}-10.23 \\
\pm 2.36\end{array}$ & $<0.001$ \\
\hline DBP (mmHg) & $\begin{array}{r}80.73 \\
\pm 1.64 \\
\end{array}$ & $\begin{array}{r}78.43 \\
\pm 0.914 \\
\end{array}$ & $\begin{array}{r}-2.30 \\
+1.79 \\
\end{array}$ & 0.208 & $\begin{array}{r}83.47 \\
\pm 1.587 \\
\end{array}$ & $\begin{array}{r}80.67 \\
\pm 1.51 \\
\end{array}$ & $\begin{array}{r}-2.80 \\
+1.85 \\
\end{array}$ & 0.141 \\
\hline FPG(mg/dl) & $\begin{array}{r}175.53 \\
\pm 11.45\end{array}$ & $\begin{array}{r}133.67 \\
\pm 6.90\end{array}$ & $\begin{array}{r}-41.87 \\
\pm 12.72\end{array}$ & 0.003 & $\begin{array}{l}160.13 \\
\pm 8.17\end{array}$ & $\begin{array}{r}115.93 \\
\pm 5.69\end{array}$ & $\begin{array}{l}-44.20 \\
\pm 8.05\end{array}$ & $<0.001$ \\
\hline HbA1c (\%) & $\begin{array}{r}8.43 \\
\pm 0.21\end{array}$ & $\begin{array}{r}7.411 \\
\pm 0.15\end{array}$ & $\begin{array}{l}-1.017 \\
\pm 0.22\end{array}$ & $<0.001$ & $\begin{array}{r}8.49 \\
\pm 0.26\end{array}$ & $\begin{array}{r}6.95 \\
+0.21\end{array}$ & $\begin{array}{l}-1.547 \\
\pm 0.22\end{array}$ & $<0.001$ \\
\hline
\end{tabular}

Weight:

There was a greater weight loss (non significant) with liraglutide $(-5.83 \pm 0.87 \mathrm{~kg}$ with liraglutide vs. $-4.2 \pm 0.46 \mathrm{~kg}$ with dulaglutide) when compared to dulaglutide $(\mathrm{p}=0.103)$. [Table $4-$ Supplementary appendix] A similar proportion of [Table 5 - Supplementary appendix] A larger proportion of patients in the liraglutide arm (13.4\%) achieved a weight loss more than $10 \%$ from baseline compared to dulaglutide (3.3\%). A highly significant weight loss of greater than $15 \%$ from baseline was seen in $6.7 \%$ patients in the liraglutide arm, but not in the dulaglutide arm. [Figure 1 - Supplementary appendix]

Blood Pressure:

There was similar reduction in DBP in both the arms $(-2.8 \pm 1.85 \mathrm{~mm}$ of $\mathrm{Hg}$ with liraglutide vs. $-2.3 \pm 1.79 \mathrm{~mm}$ of $\mathrm{Hg}$ with dulaglutide $(\mathrm{p}=0.847)$. [Table 4 -Supplementary appendix] In contrast the reduction in SBP was greater with
liraglutide $(-10.23 \pm 2.36 \mathrm{~mm}$ of $\mathrm{Hg})$ compared to dulaglutide $(-3.43 \pm 2.98 \mathrm{~mm}$ of $\mathrm{Hg})(\mathrm{p}=0.079)$, but failed to reach liraglutide $(-10.23 \pm 2.36$
statistical significance.

Dulaglutide vs. Liraglutide: Impact on the metabolic composite of HBA1C reduction AND weight loss:

The composite end point of HBA1C reduction to below $7 \%$ and a greater than $5 \%$ of body weight loss was attained by 5
(16.7\%) patients in the dulaglutide arm, while $20(50 \%)$ patients on liraglutide arm reached this composite. This $(16.7 \%)$ patients in the dulaglutide arm, while $20(50 \%)$ pate
difference was not statistically significant. $(\mathrm{p}=0.75)$. (Figure 1$)$

Adverse Events:

No serious adverse event was reported during this three month follow up. There was no hypoglycaemia reported. The the liraglutide group. Two patients complained of genital irritation, which responded to topical anti mycotics.

Discussion:

The mode of therapy co-initiating SGLT2i and GLP1RA was recently studied and published as the DURATION 8 study showing a robust weight loss and reduction in SBP in the dapagliflozin plus Exenatide-LAR arm. Strangely, this benefit did not extend to HBAlc reduction. (6) It was speculated that the rapid glucose lowering effect of SGLT-2 inhibitor Hence it would be interesting to take a look at the effect on glycaemic parameters when these two agents are added sequentially.

A couple of real-world observational data did find additive benefit of combining SGLT-2i to GLP1-RA. (8,9)

In this real world study from India, we looked at the more realistic scenario of GLP1RA therapy being instituted when oral medicines have been suboptimal and this is the first data using this combination in this order.

Both liraglutide as well as dulaglutide resulted in a significant reduction in FPG, HBA1C as well as weight from baseline. Only liraglutide not dulaglutide had a significant impact on the reduction of SBP. It should be noted here that all patients in this cohort had a reasonably well-controlled blood pressure at baseline and no antihypertensive medications were changed during the study.

This data would therefore suggest that as a first injectable, GLP1-RA is a very effective option when it is used along with a SGLT-2 inhibitor on background metformin therapy in a sequential approach.

Our study also looked at the comparative effectiveness of liraglutide versus dulaglutide as an add on option to a SGLT-2 inhibitor and metformin. There seems to be a trend favoring liraglutide vs. dulaglutide in reduction of fasting plasma glucose, HBA1C, weight and SBP. There was a statistically significant difference favoring liraglutide $63.3 \%$ with liraglutide and $30 \%$ with dulaglutide) in the proportion of patients achieving HBA1C less than 7.0\%. Larger proportion of patients achieved target HBA1C without weight gain while on liraglutide.

Off note, no serious adverse events were noted during the study.

There are several limitations in this study. Firstly it was open label and not controlled. Hence this data could not correct for numerous confounding factors that could have influenced the results. Secondly the small sample size could have for numerous confounding factors that could have influenced the results. Secondly the small sample size could have
influenced the quantum of metabolic impact. We are aware that this size was small also because only a small
percentage of our patients could afford this very expensive therapy in our "pay from pocket" setting and were "chosen" based on their ability to afford this treatment. This would also be considered a major selection bias.

\section{Conclusion:}

In this retrospective study in Indian type 2 diabetic patients poorly controlled with metformin and SGLT-2 inhibitor we found a meaningful impact of adding a GLP1-RA on all metabolic parameters. Both liraglutide and dulaglutide were effective in this regard. There were additional advantages seen with liraglutide as far achieving target HBA1C of less than 7\% and also on the quantum of weight loss and SBP reduction.

This study therefore provides a pilot and indeed generates a hypothesis supporting the sequential addition of a GLP1-RA after metformin and SGLT-2 inhibitor, in the management of $\mathrm{T} 2 \mathrm{D}$, which needs to be tested in a more systematic manner on a larger population. 